\title{
Observational Results with the 1 Meter Telescope at Yunnan Observatory During 1990-2000
}

\author{
P.S. Chen \& W.Y. Zhang \\ Yunnan Observatory \& United Laboratory of Optical Astronomy, \\ Chinese Academy of Sciences, Kunming 650011, China
}

Abstract. The specification and instruments for the 1-m telescope at Yunnan Observatory of China are briefly described in this paper. Scientific results observed during 1990-2000 are also summarized.

\section{Telescope Specification and Instrument Equipment}

\subsection{Telescope Specification}

The telescope is a Cassegrain-Ritchey-Chretien-Coude system with a $1016 \mathrm{~mm}$ primary mirror and $F / 13$. It is fully computer controlled for pointing, tracking and dome servo. The HST Guide Star Catalog is used as the reference to guide the program stars.

\subsection{Instrument Equipped}

(a) CCD Camera Format: 1024x1024 imaging pixels with a size of $24 \times 24 \mu \mathrm{m}$. Field of view in the Cassegrain focus: 6.5' x 6.5' (0." 38/pixel).

(b) Cassegrain Spectrometer (with the CCD receiver above). Focal length: $150 \mathrm{~mm}$. Spectrum range: $330-900 \mathrm{~nm}$. Dispersion: $34-195 \AA / \mathrm{mm}$.

(c) Coude spectrograph (with the CCD receiver above). Focal length: 1900 mm. Spectrum range: $330-900 \mathrm{~nm}$. Dispersion:2.8-8.4 $\stackrel{\AA}{\mathrm{A}} / \mathrm{mm}$.

\section{Main Scientific Results}

\subsection{AGNs and Blazars}

a) Optical monitoring in BVRI for over 70 BL Lac objects and Blazars. Shortterm variations (hours) have been found for 28 BL Lac objects and 18 Blazars (Jia et al. 1998; Zhang et al. 1998; Xie et al. 1999).

b) Determination of optical positions for 30 extragalactic radio sources with a mean standard error of better than $0 . " 2$ (Tang et al. 2000).

c) Long-term optical monitoring for some BL Lac objects that have shown no short-term, only long-term periodic variations. Periods have been determined for ON 231, Mkr 421 and OJ 287 (Zhang et al. 1998).

d) Optical monitoring of OJ 287 from 1993-1998. An outburst was observed in 1994-1995 (Bai et al. 1999). 
e) Photometry in BVRI and JHK for the infrared quasar IRAS 00275-2859 to determine its spectral energy distribution and absolute magnitude (Bao \& Chen 1992).

\subsection{Binary Systems and Variable Stars}

a) Light curves in B and V for many binary systems: AH Tau, FG Hya, SS Ari, AU Ser, XY Leo, BV Eri and BL Eri, YY Eri etc. (e.g. Yang et al. 1999; Liu, Q. et al. 1993,1996; Gu 1999).

b) Photometric monitoring of AU Ser has shown variation not only in the light curve but also in the color curve and found possible short period oscillations (Li et al. 1998).

c) Observations have shown the period change for some binary stars, like XY Leo and BL Eri (Liu, Q. et al. 1996; Pan et al. 1998).

d) Activity on the classical T Tau star BP Tau and its short-term variability in BVRI (Gullbring \& Chen et al. 1996).

\subsection{H $\alpha$ Observations for Chromo-spherically Active Binaries}

a) High-resolution $\mathrm{H} \alpha$ profiles, equivalent widths and radial velocities were determined for 20 chromo-spherically active binaries (Liu, X. et al. 1993, 1994).

b) High-resolution $\mathrm{H} \alpha$ observations were performed for Aur in 1989-1992. $\mathrm{H} \alpha$ profile significantly varied outside the eclipse (Cha et al. 1994).

c) Spectroscopic observations for Eri to study the magnetic field (Liu, X. et al. 1994).

\subsection{Star Rotations}

Rotational parameters for 50 bright stars were determined (Pan, K. et al. 1992).

\subsection{Objects in the Solar System}

a) Positions of 14 fragments and the brightness of 8 fragments of comet ShoemakerLevy 9 were measured three months before its collision with Jupiter (Wu et al. 1996).

b) Determination of positions for comets Hale-Bopp and Hyakutake were performed in the spring of 1996. 56 Positions derived on Feb. 28 - March 18, 1996 for Hale-Bopp were adopted by the "Minor Planets and Comets" (Wu \& Chen 1996).

c) Determination of positions of the Uranian satellites: Ariel, Titania, Oberon and Umbriel with a single measurement error of better than 0.06 (Peng et al. 1998).

\section{References}

Bai, J.M. et al. 1999, A\&AS, 136, 455

Bao, M.X., \& Chen, P.S. 1992, Acta Astronomica Sinica, 33, 337

Cha, G.W. et al. 1994, A\&A, 284, 874

Gu, S.H. 1999, A\&A, 346, 437

Gullbring, E., Chen, P.S. et al. 1996, A\&A, 307, 791 
Jia, G.B. et al. 1998, A\&AS, 128, 315

Li, Z.Y. et al. 1998, A\&AS, 131, 115

Liu, Q.Y. et al. 1993, A\&AS, 101, 253

Liu, Q.Y. et al. 1996, A\&AS, 118, 453

Liu, X.F. et al. 1994, Acta Astrophisica Sinica, 14, 53

Liu, X.F. et al. 1994, Acta Astronomica Sinica, 37, 225

Pan, K.K. et al. 1992, Acta Astronomica Sinica, 33, 32

Peng, Q.Y. et al. 1998, Chinese Journal of Space Science, 18, 91

Tang, Z.H. et al. 2000, MNRAS, 319, 717

Wu, G.J., \& Chen,P.S. et al. 1997, Acta Astronomica Sinica, 38, 183

Xie, G.Z. et al. 1999, ApJ, 522, 846

Yang, Y.L., \& Liu, Q.Y. 1999, A\&AS, 136, 139

Zhang, X. et al. 1998, Pub. Yunnan Obs., 3, 5 